\title{
FrWCSO-DRN: Fractional Water Cycle Swarm Optimizer-based deep residual network for pulmonary abnormality detection from respiratory sound signal
}

JAWAD AHMAD DAR ( $\nabla$ darjawad@rocketmail.com )

Mansarovar Global University Madhya Pradesh India https://orcid.org/0000-0001-6637-9912

Kamal Kr srivastava

Mansarovar Global University and Research Centre

Sajaad Ahmad Lone

Islamic University of Science and Technology

\section{Research Article}

Keywords: Fractional Calculus (FC), Water Cycle Algorithm (WCA), Hierarchical Attention Network, Deep Neural Network (DNN), FrWCSO-based DRN

Posted Date: November 19th, 2021

DOI: https://doi.org/10.21203/rs.3.rs-1096584/v1

License: (c) (1) This work is licensed under a Creative Commons Attribution 4.0 International License.

Read Full License

Version of Record: A version of this preprint was published at SN Computer Science on July 15th, 2022. See the published version at https://doi.org/10.1007/s42979-022-01264-0. 


\title{
FrWCSO- DRN: Fractional Water Cycle Swarm Optimizer-based deep residual network for pulmonary abnormality detection from respiratory sound signal
}

\author{
${ }^{\mathrm{a} J a w a d}$ Ahmad Dar, ${ }^{\mathrm{b}}$ Dr.Kamal Kr.Srivastava, ${ }^{\mathrm{c}}$ Dr Sajaad Ahmed Lone \\ ${ }^{a}$ Research Scholar,department of computer Science and Engineering,Mansarovar Global University \\ Madyapradesh India ORCID id : https://orcid.org/0000-0001-6637-9912 \\ Email: jawadsirphysics@gmail.com \\ ${ }^{\mathrm{b}}$ Professor,department of computer Science and Engineering,Mansarovar Global University \\ Madyapradesh India ORCID id: https://orcid.org/0000-0001-8305-7800 \\ Email:2007.srivastava@gmail.com \\ ${ }^{\mathrm{c}}$ Assistant Professor,department of computer Science and Engineering,Islamic University of Science \\ and Technology Kashmir India ORCID id: https://orcid.org/0000-0002-7089-1991 \\ Email:sajaad.lone@islamicuniversity.edu.in
}

\begin{abstract}
Respiratory sounds disclose significant information regarding the lungs of patients. Numerous methods are developed for analyzing the lung sounds. However, clinical approaches require qualified pulmonologists to diagnose such kind of signals appropriately and are also time consuming. Hence, an efficient Fractional Water Cycle Swarm Optimizerbased Deep Residual Network (FrWCSO-based DRN) is developed in this research for detecting the pulmonary abnormalities using respiratory sounds signals. The proposed FrWCSO is newly designed by the incorporation of Fractional Calculus (FC) and Water Cycle Swarm Optimizer WCSO. Meanwhile, WCSO is the combination of Water Cycle Algorithm (WCA) with Competitive Swarm Optimizer (CSO). The respiratory input sound signals are pre-processed and the important features needed for the further processing are effectively extracted. With the extracted features, data augmentation is carried out for minimizing the over fitting issues for improving the overall detection performance. Once data augmentation is done, feature selection is performed using proposed FrWCSO algorithm. Finally, pulmonary abnormality detection is performed using DRN where the training procedure of DRN is performed using the developed FrWCSO algorithm. The developed method achieved superior performance by considering the evaluation measures, namely True Positive Rate (TPR), True Negative Rate (TNR) and testing accuracy with the values of $0.963,0.932$, and 0.948 , respectively.
\end{abstract}

Keywords: Fractional Calculus (FC), Water Cycle Algorithm (WCA), Hierarchical Attention Network, Deep Neural Network (DNN), FrWCSO-based DRN

\section{Introduction}

Nowadays, world's population is suffering from different kinds of respiratory disorders [19]. According to the report of World Health Organization (WHO), the various five respiratory diseases, such as asthma, tuberculosis, acute lower respiratory tract infection (LRTI), lung cancer, and chronic obstructive pulmonary disease (COPD) may leads to the demise of more than 3 million people around the world [4]. In addition, People are getting out to particulate issue while working in defectively ventilated work areas and also come in contact with tiny substances coming from vehicles, toxic gaseous emission from the chimneys of industries and chemical plants. All these factors are responsible for the growth of lung related diseases [20] [19]. Respiratory sounds coming from airways and lungs offer imperative information about their pathologies and physiologies [22]. Moreover, the respiratory sounds are generally classified into two classes, namely abnormal sound and normal sound with respect to the 
acoustic respiratory lung sound coming from lungs [23]. Furthermore, various kinds of respiratory problems are progressive in nature and hence worsen the respiratory potential of the human lungs. Inspite of the phenomenal advancements in the areas of medical science, identification of various diseases associated to lungs at premature stage is still a topic of concern. The abnormalities coming from the lungs are utilized for distinguishing abnormal and normal pulmonary sounds [26].

Pulmonary sounds are generally classified into adventitious sounds and normal breath sounds and are heard on the chest wall and mouth. Breath sounds which are considered as normal respiratory noises are synchronous with the flow of air through the airways from laminar to turbulent with a frequency spectrum of $200-600 \mathrm{~Hz}$ in powerful and healthy lungs. Besides, the crackles with a frequency spectrum of 100 to $2000 \mathrm{~Hz}$ and a time period of less than 70 ms represent an important element of adventitious sounds. They are frequently attributed to the bubbling of secretions in the airways or to the impulsive alterations in gas pressure in the minute airways. Moreover, the crackles are generally categorized as coarse and fine. In pulmonary sounds, Timing, pitch, and number of crackles reflect the type and phase of disease [1]. For the patients with bronchiectasis and chronic air flow obstruction, short pitched crackles called coarse crackles are created while the crackles of interstitial fibrosis are fine or high pitched and take place in a mid to late inspiration. The application of signal processing techniques to the data attained through the stethoscope enabled the analysis of lung sounds simpler, objective and also makes the non-invasive auscultation approach more valuable in the pulmonary disease analysis. Recently, numerous researchers have been conducted to acquire the parametric representations of lung sounds for creating a more objective basis for their assessment [2].

With the expansion of respiratory lung disorders [33], more consideration is being paid on the risk-free diagnosis based on assessment of lung sounds which contains plentiful information about the lung condition. However, the auscultation of lung sounds has a very restricted practice due to the insufficiency of information it can attain, its reliance on physician's knowledge, and its improved rate of misdiagnosis. Hence, it is necessary to establish a proficient technique for identifying lung sounds and also for analyzing related diseases based on the investigation of the more useful lung sounds composed by electronic stethoscope and expands an effective way for identifying lung sounds and its associated diseases [27]. During diagnosis practice, it is supportive for precisely judging the characteristic vectors and for evaluating the disease reasons to de-noise abstract vibration frequency and lung sounds, amplitude gradient and sound waves amplitude [27]. Moreover, clinical respiratory sounds are complicated to get in practice, and the samples of lung sounds are small. Thus, the researchers have normally selected conventional machine learning techniques, such as hidden Markov model (HMM) [29], support vector machine (SVM) [30] artificial neural network (ANN) [28], and k-Nearest Neighbor [21], instead of a deep learning technique for categorizing the lung sounds [2].

The key objective of this research is to introduce a robust technique for pulmonary abnormality detection named FrWCSO algorithm. The respiratory sound signal is preprocessed and the features, such as Bark Frequency Cepstral Coefficient (BFCC), shortterm features, statistical features, and wavelet transform are extracted to achieve pulmonary abnormality detection. Here, the data augmentation process is performed using Window Warping (WW), jittering, and cropping. Once data augmentation is done, feature selection is performed using proposed FrWCSO. Moreover, the DRN classifier is accomplished for pulmonary abnormality detection and the training practice of DRN is carried out using the 
proposed optimization algorithm, named FrWCSO algorithm, which is the hybridization of FC and WCSO. On the other hand, WCSO is the integration of WCA and CSO, respectively.

The major goal of the research work is elucidated as follows:

* Developed FrWCSO-based DRN: An effective and robust optimization algorithm, called FrWCSO is developed for feature selection and also for training the DRN classifier for attaining better pulmonary abnormality detection results. However, FrWCSO is the incorporation of FC and WCSO, which is the integration of WCA and CSO.

The arrangement of the research paper is prepared as follows, section 2 reviews the exiting pulmonary abnormality detection methods, the proposed pulmonary abnormality detection model is portrayed in section 3, the implementation outcomes are presented in section 4, and the conclusion of the paper is described in section 5 .

\section{Motivations}

The various existing pulmonary abnormality detection techniques based on the respiratory sound signals along with its advantages and disadvantages are illustrated in this section that encourage the researchers to design the proposed FrWCSO-based DRN method.

\subsection{Literature Survey}

This section reviews the various eight existing pulmonary abnormality detection methods with its advantages and disadvantages.

Alfonso Monaco et al. [1] introduced a multi-time-scale machine learning model to categorize the respiratory sounds like crackles and wheezes. This model comprises three different modules, such as data standardization, multi-time-scale feature extraction, and classification module. This technique was utilized for discriminating the well controls from the patients with respiratory disorders. This model was more appropriate for large-scale applications, but this method failed to identify the important sounds at the respiratory cycle level. Fei Meng et al. [2] developed a machine learning method to recognize the respiratory sounds using wavelet coefficients. This method integrated the idea of wavelet signal similarity with relative wavelet energy and the wavelet entropy. This approach attained improved classification accuracy. On the other hand, the normalization module may leads to an increase in errors. JyotibdhaAcharyay, and Arindam Basuyyy [3] presented a Deep Convolutional Neural Network-Recurrent Neural Network (CNN-RNN) model to classify the respiratory sounds using Mel spectrograms. Here, a local log quantization of trained weights is accomplished for minimizing the memory requirements. This model obtained substantial weight compression without any adjustments in the architectural model. Meanwhile, this technique failed to reduce the operational cost. Samiul Based Shuvo et al. [4] introduced a light weight $\mathrm{CNN}$ model for categorizing the respiratory diseases withhybrid scalogram features. This model achieved improved accuracy in categorizing the ternary chronic diseases, but the major challenge lies in utilizing this approach in the real-world clinical applications.

M. Fraiwan et al. [5] introduced a deep learning framework using CNN and bidirectional long short-term memory (LSTM) for classifying the respiratory sounds. This deep learning model achieved improved performance in categorizing the lung sounds. Meanwhile, the training structure and the pre-processing techniques were not adjusted in order to achieve effective results. Neeraj Baghel et al. [6] designed an automatic classification approach based 
on machine learning for diagnosing multiple pulmonary diseases from lung sounds. This method effectively minimized the computational time in treating the pulmonary diseases and this method was more appropriate for real-time applications. However, this method failed to reduce the computational overheads. Sibghatullah I. Khan et al. [7] modeled an empirical mode decomposition (EMD) method for extracting the intrinsic mode functions (IMFs) of lung sounds because of its non-linear and non-stationary nature. However, this method failed to consider novel deep learning classifiers for improving the performance of classification. S.Jayalakshmy and Gnanou Florence Sudha [8] presented a pre-trained optimized Alexnet $\mathrm{CNN}$ model to predict the respiratory disorders. This model achieved enhanced performance in classifying the respiratory disorders, thereby preventing ambiguous analysis. Moreover, this method failed to consider larger and heterogeneous datasets for effective classification results.

\subsection{Challenges}

The several issues faced by various abnormality classification techniques based on the respiratory sound signals are elucidated as follows,

- The major constraint in multi-scale machine learning model introduced in [1] is the lack of capability for detecting the major sounds at the respiratory cycle phase. In addition, the deep learning methods, namely Long Short-Term Memory (LSTM) and ResNet were not considered for enhancing the classification performance.

- The machine learning method developed in [2] attained superior performance, but this method failed to incorporate the respiratory sounds with different medical parameters, like spirometry parameters for providing intellectual disease recognition model.

- In [5], deep learning method was designed for categorizing the respiratory sounds. However, this work can be further extended by enlarging the dimensions of the dataset for including numerous subjects and a wider collection of diseases.

- An automatic classification approach was introduced for diagnosing respiratory problems, but this method failed to recognize other types of lung sounds associated to various respiratory disorders [6].

- In [8], an adaptive kernel selection algorithm was designed to classify the lung sounds of humans. This algorithm effectively classified the normal and abnormal lung sounds. However, this algorithm does not classify the biomedical sound signals, namely phonocardiogram and bowel sounds in the abnormal and normal category.

\section{Proposed FrWCSO-based DRN for pulmonary abnormality detection}

This section illustrates the process of designing and developing an approach for the detection of pulmonary abnormality with the respiratory Sound signal. The various phases involved in detecting the pulmonary abnormalities are, pre-processing phase, feature extraction phase, data augmentation phase, feature selection phase, and detection phase. Initially, the input respiratory sound signal is fed to the pre-processing phase, which is done based on Hanning window [13] and spectral gating-based noise reduction method [14] for removing the unwanted distortions and external calamities present in the input signal. Once the preprocessing is done, the feature extraction process is carried out for extracting the features, such as BFCC [15] and short term features like spectral flux, spectral centroid, and Power Spectral density (PSD) [16]. In addition, the statistical features, like mean, standard deviation, entropy, energy, kurtosis, and wavelet transform [17] are extracted effectively from the input signal. Once the appropriate features are extracted, the data augmentation is 
done for enlarging the dimensions of the dataset. After that, the feature selection process is performed using the proposed FrWCSO. Moreover, the proposed FrWCSO is newly designed by integrating FC [10] and WCSO algorithm. Meanwhile, WCSO is the combination of WCA [11] and CSO [12]. Finally, the pulmonary abnormality detection is performed using DRN [9] that is trained using the proposed FrWCSO for achieving effective detection outcomes. Figure 1 depicts the schematic view of the developed FrWCSO-based DRN for pulmonary abnormality detection.

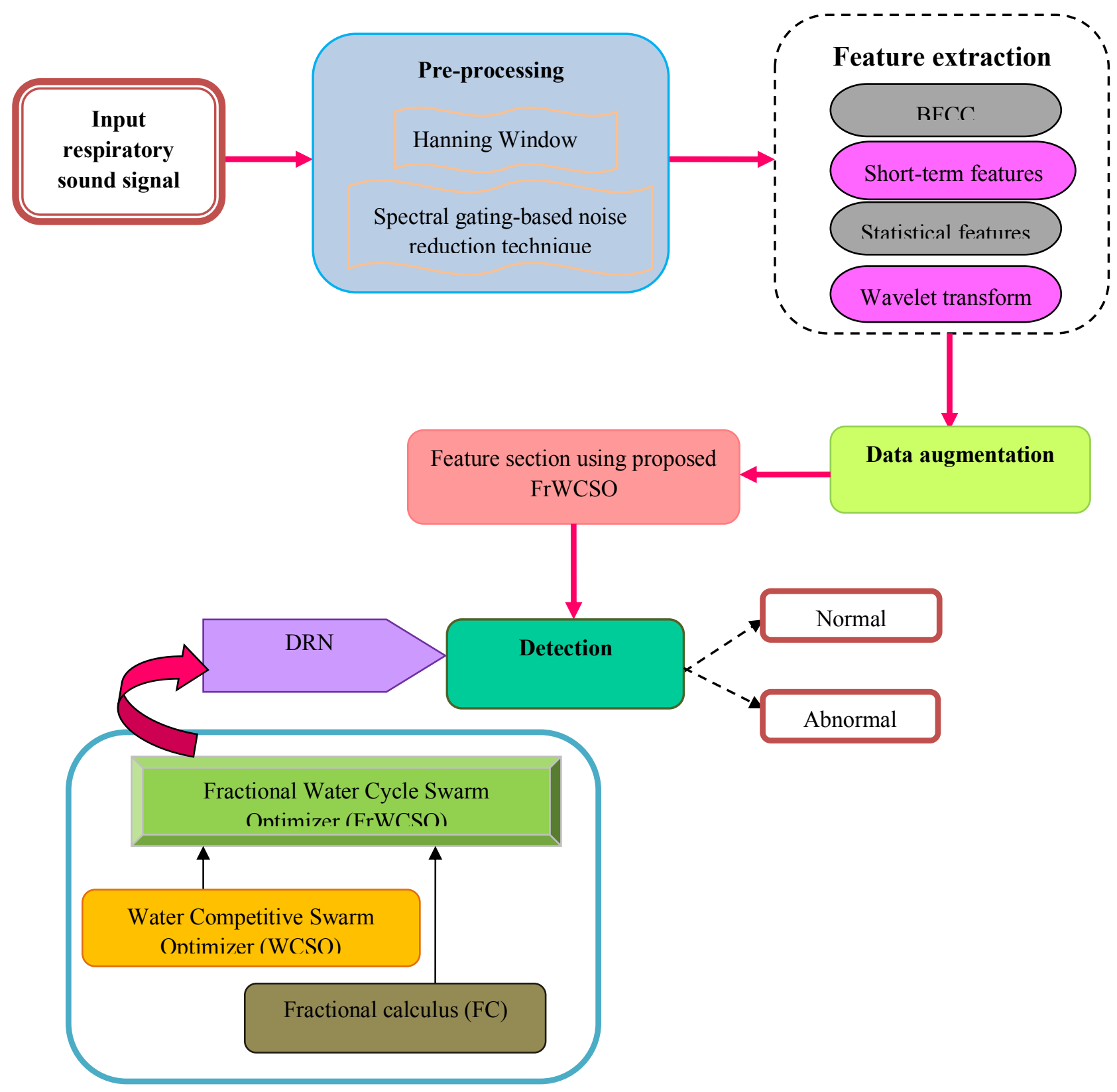

Figure 1.Schematic diagram of developed FrWCSO-based DRN for detecting pulmonary abnormality. 


\subsection{Input acquisition}

At first, the input respiratory sound signal is acquired from a dataset $S$ with $n$ number of signals, which is given as,

$$
S=\left\{H_{1}, H_{2}, \ldots H_{g}, \ldots H_{n}\right\}
$$

where, $n$ represents the overall respiratory sound signals, and $H_{g}$ denotes the $g$ input signal, which is fed as an input to the pre-processing phase.

\subsection{Pre-processing}

Once the input respiratory sound signals are acquired, pre-processing is performed using Hanning window [13] and Spectral gating-based noise reduction technique [14] to remove the distortions from the signals. The pre-processing phase is more effectual in making the further phases more viable. The Hanning window and Spectral gating-based noise reduction technique is illustrated below as follows,

\subsubsection{Hanning window}

Hanning window [13] or Hann window is an approach applied for removing the noises or distortions from the input respiratory sound signals. This type of technique is employed as a pre-analysis module for improving the results. The infinite streams of the sound signals are transformed to incessant streams of blocks of samples known as frames. The frames extracted from the respiratory sound signals are windowed using Hanning window technique to eliminate the unnecessary signals for collecting the valuable information. This approach enables the smoothing without losing any contents present at the sharp points or edges. Moreover, the Hann window function is represented as,

$$
H(Z)= \begin{cases}0.5\left(1-\cos \left[\frac{2 \pi t}{G-1}\right]\right) \cong \sin ^{2} \frac{\pi t}{(G-1)} & ; 0 \leq t \leq G-1 \\ 0 & ; \text { Otherwise }\end{cases}
$$

where, $H(Z)$ denotes the Hann window function.

\subsubsection{Spectral gating-based noise reduction method}

The spectral gating-based noise reduction method [14] is an algorithm accomplished for removing the unnecessary noises from the signals. The various steps of spectral gating-based noise reduction algorithm are presented below as follows,

Step 1: A Fast Fourier Transform (FFT) is measured over the noise audio clip

Step 2: Statistics are measured over FFT of the noise relating to frequency

Step 3: A value of threshold is computed with respect to the noisy statistics and the chosen sensitivity of the algorithm

Step 4: An FFT is measured over the signal 
Step 5: A mask is evaluated by comparing the FFT of the signal to the value of threshold

Step 6: The smoothing of mask with a filter is done in terms of time and frequency

Step 7: The mask is applied to the FFT of the signal, and finally the signal is inverted

Accordingly, the pre-processed output generated using the Hanning window and the Spectral gating-based noise reduction technique is denoted as $P_{p}^{*}$, which is given to the feature extraction phase.

\subsection{Feature extraction}

Once the pre-processing is done, the feature extraction process is carried out to extract the significant features as the feature extraction step is very much significant for detecting the pulmonary abnormalities more effectively. However, the features, such as BFCC, short-term features, statistical features, and wavelet transform features gives required information regarding the abnormalities in the respiratory sound signals. Furthermore, the various extracted features are explained below as follows,

\subsubsection{BFCC}

The BFCC [15] representation is used for extracting the significant features from input respiratory sound signal. BFCC distorts power spectrum so that it matches with individual intensity observations. The frequency bands of BFCC are linear to $500 \mathrm{~Hz}$ and the equation is expressed as,

$$
\operatorname{Bark}(c)=13 \arctan (0.00076 c)+3.5 \arctan \left(\left(\frac{c}{7500}\right)^{2}\right) \text { bark }
$$

where, Bark denotes the bark frequency, and $c$ specifies the frequency in Hertz. The BFCC feature output is represented as $f_{1}$ with the dimension $[920 \times 200]$.

\subsubsection{Short term features}

Some of the short-term features, like spectral flux, spectral centroid, and PSD are illustrated below as follows,

\section{a) Spectral flux}

Spectral flux is a feature which computes the rate of spectral information in the respiratory sound signal. The information is measured based on the frequency-driven parameters so that the difference value among the successive spectral frames is also computed. The equation for spectral flux feature is givenas,

$$
t_{1}=\sum_{V=0}^{m / 2-1}\left(\left|J_{r}(U)\right|^{2}-\mid J_{r-1}(U)^{2}\right)
$$

where, the term $J_{r}(U)$ indicates the spectrum value of the respiratory sound signal, and $t_{1}$ denotes the spectral flux feature with the dimension $[920 \times 1]$.

\section{b) Spectral centroid}


Spectral centroid feature defines the spectrum center gravity and it measures the spectral values and higher centroid values related to enhanced signal frequency. Moreover, this feature provides the information regarding thesignal variations and is expressed as,

$$
t_{2}=\frac{\sum_{s=1}^{\rho / 2} T(s) B_{l}(s)}{\sum_{s=1}^{\rho / 2} B_{l}(s)}
$$

where, $B_{l}(s)$ denotestheshort-time Fourier transform, $T(s)$ indicates the frequency of $s^{\text {th }}$ frame, the frame length is represented as $\rho$, and the spectral centroid feature is signified as $t_{2}$ with the dimension $[920 \times 1]$.

\section{c) PSD}

PSD [16] is a feature which is used for stationary signal processing and is more appropriate for narrowband signals. It is a general signal processing method that distributes the signal power over frequency and exhibits the strength of the energy as the frequency function. However, the equation for PSD is expressed as,

$$
t_{3}=\frac{1}{N y q} * \sum_{\aleph=1}^{N y q}|F F T|^{2}
$$

where, Nyq indicates the Nyquist frequency, $\aleph$ denotes the frequency in hertz, and the PSD feature is specified as $t_{3}$ with the dimension $[920 \times 12]$.

The extracted features, namely spectral flux, spectral centroid, and PSD are integrated to form a short-term feature $f_{2}$.

$$
f_{2}=\left\{t_{1}, t_{2}, t_{3}\right\}
$$

\subsubsection{Statistical features}

Some of the statistical features extracted in the pulmonary abnormality detection process are, mean, standard deviation, entropy, energy, and kurtosis and are explained below as follows,

a) Mean: It is the feature that defines the average of sum of all the vector values and is expressed as,

$$
s_{1}=\frac{1}{d} \sum_{r=1}^{d} W_{r}
$$

where, $W_{r}$ signifies vector values of every class, $d$ denotes the total vector values of preprocessed signal, and mean feature is denoted as $s_{1}$ having the dimension $[920 \times 1]$.

b) Standard deviation: Standard deviation is a feature that quantifies the total variations in the vector values, which is signified as $s_{2}$ with the dimension $[920 \times 1]$. 
c) Entropy: Entropy is a measure which computes the signal vectors with higher amount of information. The information at the corner and edge is measured using the entropy feature $s_{3}$ having the dimension $[920 \times 1]$.

d) Energy: Energy feature of a pre-processed signal is computed by summing the vector values present in the signal and is denoted as with the dimension $[920 \times 1]$.

e) Kurtosis: Kurtosis defines the sharpness of curve in a frequency distribution, which is represented as $s_{5}$ with the dimension $[920 \times 1]$.

The features extracted using statistical features, like mean, standard deviation, entropy, energy, and kurtosis are incorporated together to form a feature $f_{3}$.

$$
f_{3}=\left\{S_{1}, s_{2}, S_{3}, s_{4}, s_{5}\right\}
$$

\subsubsection{Wavelet transform feature}

A wavelet [17] is a wave-like vibration accompanied by amplitude that increases, decreases, and repeats around zero. Wavelets have valuable and practical features for signal processing. Wavelets are also useful for wavelet-based decompression algorithms designed to reduce loss and restore unprocessed information. In addition, wavelet transform can be considered as a time-frequency representation of distinctive signals that are utilized to model systems, signals and processes through an incorporation of numerous wavelet functions. Moreover, the wavelet transform is represented as an arbitrary waveform after being scaled. To perform scaling, one small waveform is utilized as a pattern to be enlarged, transited, and reduced. Thus, the wavelet feature is indicated as $f_{4}$ with the dimension $[920 \times 200]$.

Once all the significant features are extracted, the extracted features are incorporated to generate a final feature vector output represented as $F_{e}^{*}$.

$$
F_{e}^{*}=\left\{f_{1}, f_{2}, f_{3}, f_{4}\right\}
$$

Where, $f_{1}$ signifies the BFCC feature, the short-term features are represented as $f_{2}, f_{3}$ specifies the statistical feature, $f_{4}$ denotes the wavelet transform feature, and $F_{e}^{*}$ indicates the feature extraction output with the dimension [920×419], which is then presented as an input to the data augmentation.

\subsection{Data augmentation}

Data augmentation defines a process that augments the dataset by establishing unobserved samples. The major aim of data augmentation is done to improve the pulmonary abnormality detection performance and also in enlarging the dimensions of the dataset. Lesser number of training samples may lead to over fitting. To avoid over-fitting problems during training, the following data augmentation methods, such as WW, jittering [18], and cropping are used and are described below as follows,

a) Window warping (WW): WW [18] is a time-series-specific approach, which aims at maximizing the quantity and the diversity of the data. It selects a slice of the time series at random using a sliding window in and warps it by dilating or squeezing procedure. The choice of the warping area is significant for the performance of the $\mathrm{WW}$, because the time 
scale has important physiological meanings. Hence, the WW method is two-sided from time to time. It may establish non-redundant samples to the dataset with accurate parameter selection. However, if the warping area or warping ratio is not carefully chosen, the generated new samples may devastate the physiological importance and the detection performance.

b) Jittering: Jittering [18] is a scheme that adds jitter on the signal recordings at random to imitate the noise. In addition, Jittering adds unnecessary noise to the recordings which are previously dicarded from baseline or noise.

c) Cropping: Cropping is done to crop or select the specific region from the signals.

As a result, the data augmentation result generated using WW, Jittering, cropping method is signified as $A_{p}^{*}$ with the dimension $[3680 \times 419]$, which is fed as an input to the feature selection phase.

\subsection{Feature selection}

Reducing the overall features is important in addressing the computational complexity problems. Feature selection process is performed using the proposed FrWCSO algorithm. Here, the developed FrWCSO algorithm trains the classifier weights in order to achieve effective optimal solution. The hybridization WCSO with the FC algorithm exhibits the efficiency of the developed scheme by minimizing the computational complexity issues in an enhanced way. Moreover, the representation of solution encoding and the computation of fitness measure are described below as follows,

Solution encoding: Solution encoding is a depiction of solution vector that selects the optimal features in order to achieve optimal solution. The dimension of solution is given as $[1 \times w]$, where $w$ denotes total number of features to be selected. Figure 2 portrays solution encoding.

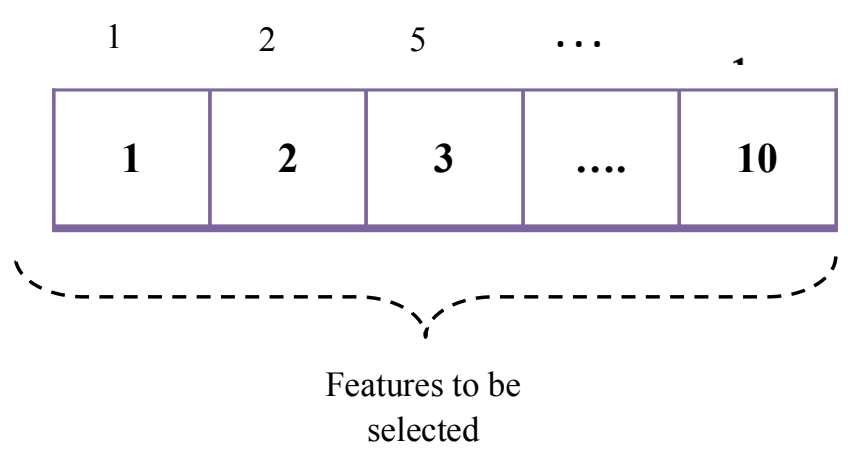

Dimension of solution

Fitness measure: The fitness measure is used for identifying the optimal solution by computing the optimal value of fitness and the equation for fitness measure is given as,

$$
\frac{R V\left(f_{i}, \ell\right)+C S\left(f_{i}, \ell\right)}{2}
$$

where, $R V$ and $C S$ signifies the RV coefficient and cosine similarity between $i^{\text {th }}$ feature and class label.

The algorithmic phases of the developed FrWCSO algorithm is elucidated below as follows, 
a) Initialization: The population is initialized with $N$ number of raindrops, and is given as,

$$
Y=\left\{Y_{1}, Y_{2}, \cdots, Y_{k}, \cdots Y_{N}\right\} ; 1 \leq k \leq N
$$

where, $N$ signifies the overall raindrop solutions, and $Y_{k}$ denotes the $k^{\text {th }}$ raindrop.

b) Calculate fitness measure: The fitness measure is computed by the equation expressed in (11).

\section{c) Compute the cost function:}

The decision variables $\left(Y_{1}, Y_{2}, \ldots, Y_{N_{\text {var }}}\right)$ can be indicated as the floating-point values or can be represented as a predefined set for the discrete and continuous limits. The cost of a raindrop is computed using the cost function evaluation which is given as,

$$
A_{e}=\operatorname{Cost}_{e}=g\left(Y_{1}^{e}, Y_{2}^{e}, \ldots, Y_{N_{\mathrm{var}}}^{e}\right) e=1,2,3, \ldots, N_{p o p}
$$

where, the total number of raindrops is signified as $N_{p o p}$ and $N_{\text {var }}$ represents the total number of design values. For the main phase, $N_{p o p}$ raindrops are created. In addition, $N_{s r}$ from the optimum least values are considered as sea and rivers. Here, $N_{s r}$ specifies the summing up of the overall rivers, and the individual sea. The residual raindrops forms the streams to pass into the sea or rivers, and the equation is given as,

$$
\begin{gathered}
N_{s r}=\text { Total number of rivers }+\underset{\text { sea }}{1} \\
N_{\text {Raindrops }}=N_{p o p}-N_{s r}
\end{gathered}
$$

d) Compute the intensity of flow: The raindrops can be allocated to the sea and the river on the basis of intensity of flow, and the equation for computing the intensity of flow is expressed as,

$$
N_{q}=\text { round }\left\{\left|\frac{\operatorname{Cost}_{q}}{\mid \sum_{e=1}^{N_{s r}} \operatorname{Cos} t_{e}}\right| \times N_{\text {Raindrops }}\right\} q=1,2, \ldots . N_{s r}
$$

where, $N_{q}$ denotes the streams passing to particular sea or rivers.

e) Evaluate the flow of stream to the river or sea: The streams are formed from every raindrop, and then join with each other to produce fresh rivers. Besides, the streams run to the sea, and every streams and rivers connects in sea. The streams passes to the river along the linking line between them based on the randomly selected distance and the equation is expressed as,

$$
Y \in(0, A \times k), \quad A>1
$$

where, the value of $A$ lies within the range of 1 and 2 , the present distance among the river and stream is signified as $k$. The value of $A$ being superior to one enables the streams to pass 
in various directions over the rivers. In addition, this kind of rule can be utilized in passing rivers to sea. Hence, the latest position of rivers and streams is expressed as,

$$
Y_{\text {river }}^{z+1}=\frac{1-Q_{2}-\varphi Q_{3}}{\operatorname{rand} \times M-Q_{2}-\varphi Q_{3}}\left[\operatorname{rand} \times M Y_{\text {sea }}^{z} \frac{-Q_{1} X^{z}+Q_{2} Y_{x}^{z}+\varphi Q_{3} \bar{Y}}{1-Q_{2}-\varphi Q_{3}}(1-\text { rand } \times M)\right]
$$

Substituting $Y_{\text {river }}^{z}$ on both sides,

$$
Y_{\text {river }}^{z+1}-Y_{\text {river }}^{z}=\frac{1-Q_{2}-\varphi Q_{3}}{\text { rand } \times M-Q_{2}-\varphi Q_{3}}\left[\text { rand } \times M Y_{\text {sea }}^{z} \frac{-Q_{1} X^{z}+Q_{2} Y_{x}^{z}+\varphi Q_{3} \bar{Y}}{1-Q_{2}-\varphi Q_{3}}(1-\text { rand } \times M)\right]-Y_{\text {river }}^{z}
$$

Applying FC,

$$
\begin{aligned}
& D^{\hbar}\left[Y_{\text {river }}^{z+1}\right]=\frac{1-Q_{2}-\varphi Q_{3}}{\text { rand } \times M-Q_{2}-\varphi Q_{3}}\left[\text { rand } \times M Y_{\text {sea }}^{z} \frac{-Q_{1} X^{z}+Q_{2} Y_{x}^{z}+\varphi Q_{3} \bar{Y}}{1-Q_{2}-\varphi Q_{3}}(1-\text { rand } \times M)\right]-Y_{\text {river }}^{z} \\
& Y_{\text {river }}^{z+1}-\hbar Y_{\text {river }}^{z}-\frac{1}{2} \hbar Y_{\text {river }}^{z-1}-\frac{1}{6}(1-\hbar) Y_{\text {river }}^{z-2}-\frac{1}{24} \hbar(1-\hbar)(2-\hbar) Y_{\text {river }}^{z-3}=\frac{1-Q_{2}-\varphi Q_{3}}{\text { rand } \times M-Q_{2}-\varphi Q_{3}} \\
& {\left[\text { rand } \times M Y_{\text {sea }}^{z} \frac{-Q_{1} X^{z}+Q_{2} Y_{x}^{z}+\varphi Q_{3} \bar{Y}}{1-Q_{2}-\varphi Q_{3}}(1-\text { rand } \times M)\right]-Y_{\text {river }}^{z}} \\
& Y_{\text {river }}^{z+1}=\hbar Y_{\text {river }}^{z}+\frac{1}{2} \hbar Y_{\text {river }}^{z-1}+\frac{1}{6}(1-\hbar) Y_{\text {river }}^{z-2}+\frac{1}{24} \hbar(1-\hbar)(2-\hbar) Y_{\text {river }}^{z-3}+\frac{1-Q_{2}-\varphi Q_{3}}{\text { rand } \times M-Q_{2}-\varphi Q_{3}} \\
& {\left[\text { rand } \times M Y_{\text {sea }}^{z} \frac{-Q_{1} X^{z}+Q_{2} Y_{x}^{z}+\varphi Q_{3} \bar{Y}}{1-Q_{2}-\varphi Q_{3}}(1-\text { rand } \times M)\right]-Y_{\text {river }}^{z}} \\
& Y_{\text {river }}^{z+1}=Y_{\text {river }}^{z}(\hbar-1)+\frac{1}{2} \hbar Y_{\text {river }}^{z-1}+\frac{1}{6}(1-\hbar) Y_{\text {river }}^{z-2}+\frac{1}{24} \hbar(1-\hbar)(2-\hbar) Y_{\text {river }}^{z-3}+\frac{1-Q_{2}-\varphi Q_{3}}{\text { rand } \times M-Q_{2}-\varphi Q_{3}} \\
& {\left[\text { rand } \times M Y_{\text {sea }}^{z} \frac{-Q_{1} X^{z}+Q_{2} Y_{x}^{z}+\varphi Q_{3} \bar{Y}}{1-Q_{2}-\varphi Q_{3}}(1-\text { rand } \times M)\right]}
\end{aligned}
$$

where, rand denotes the uniformly distributed random number between 0 and 1 , $Q_{1}, Q_{2}, Q_{3} \in[0,1]^{n}, X^{z}$ denotes the velocity of loser, $Y_{x}^{z}$ implies the location of winner, the value of $N$ lies among the range 1 and 2, $\varphi$ represents the parameter that controls the influence of $\bar{Q}(u)$, the mean position value of every particles in $v(u)$ is signified as $\bar{Q}(u)$, and $\hbar$ lies between the range 1 and 2 , 
f) Determine the condition for evaporation: Evaporation is the key factor that protects the process from immature convergence. Here, the clouds are created by taking the evaporated water into the atmospheric region, and thereafter condenses in the colder atmosphere so that the water gets released back in the form of rain to the earth. The rain drops create new streams passing to rivers, which then flows into the sea. Moreover, $h_{\max }$ represents the minimum number closer to 0 and the value of $h_{\max }$ adaptively decreases as,

$$
h_{\max }^{e+1}=h_{\max }^{e}-\frac{h_{\max }^{e}}{\text { maxiteration }}
$$

g) Raining procedure: After the completion of evaporation process, the raining process is performed. Here, the new raindrops forms the streams in various places, and the position of the newly formed streams is expressed as,

$$
R_{\text {stream }}^{\text {new }}=l b+\operatorname{rand} \times(u b-l b)
$$

where, $l b$ denotes lower bound, and $u b$ implies the upper bounds.

$$
R_{\text {stream }}^{\text {new }}=R_{\text {sea }}+\sqrt{\mu} \text { rand } k\left(1, Z_{\mathrm{var}}\right)
$$

where, $\mu$ signifies the coefficient that represents the searching area limit closer to the sea and the value is set to 0.1 , rand $k$ implies the frequently distributed random number.

h) Feasibility assessment: The feasibility evaluation is done for finding the optimal value. If the newly obtained solution has the optimal value, then the existing one is replaced with the optimal value.

i) Termination: All the above mentioned phases are iteratively performed until the finest solution is achieved. Table 1 presents the pseudo code of proposed FrWCSO algorithm.

Table 1.Pseudo code of proposed FrWCSO algorithm

\begin{tabular}{|c|l|}
\hline SI. No & \multicolumn{1}{|c|}{ Pseudo code of developed FrWCSO algorithm } \\
\hline 1 & Begin \\
\hline 2 & \multicolumn{1}{|c|}{ Initialize $N_{s r}, k_{\max }, N_{p o p}$} \\
\hline 3 & Create initial population randomly by equation (12) \\
\hline 4 & \multicolumn{1}{|c|}{ Compute the cost of every raindrop by equation (13) } \\
\hline 5 & Calculate the intensity of flow using equation (16) \\
\hline 6 & Estimate the flow of stream to the rivers using equation (23) \\
\hline 7 & for \\
\hline 8 & if $A>1$ \\
\hline 9 & Satisfy evaporation condition \\
\hline 10 & else \\
\hline 11 & Replace the position of the river with sea \\
\hline 12 & end if \\
\hline 13 & if $\left|N_{\text {sea }}^{e}-N_{\text {river }}^{e}\right|<k_{\max }$ \\
\hline 14 & Replace the location of river with sea \\
\hline 15 & else \\
\hline
\end{tabular}




\begin{tabular}{|l|l|}
\hline 16 & Satisfy evaporation circumstance \\
\hline 17 & end if \\
\hline 18 & Initiate raining procedure using equation (25) and equation (26) \\
\hline 19 & Minimize the value of $k_{\max }$ by equation (24) \\
\hline 20 & Validate the convergence criteria \\
\hline 21 & Satisfies the convergence criteria \\
\hline 22 & Stop \\
\hline 23 & else \\
\hline 24 & Repeat the process \\
\hline 25 & end for \\
\hline 26 & end \\
\hline
\end{tabular}

As a result, the feature selection output is denoted as with dimension [ $3680 \times 100]$, and is fed as an input to the data augmentation phase.

\subsection{Proposed FrWCSO-based DRN for pulmonary abnormality detection}

The feature selection output $F_{p}^{*}$ is subjected as an input to the final phase called pulmonary abnormality detection. Here, the DRN classifier [9] is utilized for detecting the pulmonary abnormalities as this classifier achieves better generalization performance and accuracy. Meanwhile, the training practice of DRN classifier is performed using the developed FrWCSO algorithm. Furthermore, the architectural representation and training process of DRN are described below as follows

\subsubsection{Architecture of DRN}

The DRN model [9] comprise of various layers, such as input layer, convolutional (Conv) layer, batch normalization, activation function, average pooling layer, flatten layer, and dense layer. Here, each layer considers the output of the previous layer to execute the further process.

\section{Conv layer:}

During the training process, the two-dimensional conv layer minimizes the free parameters and the input is processed using a sequence of filters known as kernel with local connections. The mathematical operation of conv layer is a dot product of input and the kernel for sliding every filter on the input matrix. However, the computation process of conv layer is expressed as,

$$
\begin{aligned}
& \operatorname{Conv} 2 d(d)=\sum_{w=0}^{u-1} \sum_{h=0}^{u-1} L_{w, h} \bullet d_{(i+w),(j+h)} \\
& \operatorname{Conv1d}(d)=\sum_{b=0}^{\omega_{i n}-1} L_{b} * d
\end{aligned}
$$


where, $d$ signifies the 2-D convolutional output from the preceding layer, $i$ and $j$ records the coordinates, $L$ implies $u \times u$ kernel matrix, and $w$ and $h$ indicates kernel matrix index. Hence, $L_{b}$ signifies the size of the kernel for $b^{\text {th }}$ input neuron, and $*$ symbolizes the cross correlation operator.

\section{Average pooling layer:}

The pooling layer is generally attached into the conv layers, and is operated on every slice and depth of feature maps for minimizing the spatial dimensions of feature maps in order to control the over fitting problems.

$$
\begin{aligned}
& w_{\text {out }}=\frac{w_{\text {in }}-b_{w}}{\theta}+1 \\
& h_{\text {out }}=\frac{h_{\text {in }}-b_{h}}{\theta}+1
\end{aligned}
$$

where, the height and width of input matrix is signified as $h_{\text {in }}$ and $w_{\text {in }}$, whereas $h_{\text {out }}$ and $w_{\text {out }}$ are the output. In addition, the height and width of the kernel size is represented as $b_{h}$ and $b_{w}$.

\section{Activation function:}

In order to enhance the non-linearity of the extracted features, non-linear activation function known as Rectified Linear Unit (ReLU) is utilized by learning the non-linear and complicated features. However, the ReLU function is expressed as,

$$
\eta(d)=\left\{\begin{array}{l}
0 ; d<0 \\
d ; d \geq 0
\end{array}\right.
$$

where, $d$ represents the inputs feature.

\section{Residual blocks:}

Residual blocks possess a shortcut association from input to output so that the inputs are connected to the output when the output and input are of equal size. Meanwhile, the dimension matching factor is accomplished to match the dimensions of input and output.

$$
\begin{aligned}
& x=\mathfrak{R}(d)+d \\
& x=\mathfrak{R}(d)+W_{d} d
\end{aligned}
$$

where, $d$ signifies the input of residual blocks, $x$ denotes the output of residual blocks, $\mathfrak{R}$ indicates the mapping relationship among the input and the output dimension, and $W_{d}$ specifies the dimension matching factor.

\section{Linear classifier:}

Linear classifier is constructed based on the integration of softmax function and fully connected (FC) layer. Here, each neuron is connected from one layer to other layer using FC 
layer, whereas the softmax function is utilized for normalizing the input vector to probability vector in such a way that the class having highest probability is selected as the final computed output.

$$
\begin{array}{r}
x=W_{O \times P} d_{P \times Q}+e_{O \times Q} \\
\varepsilon\left(d_{\ell}\right)=\frac{m^{d_{\ell}}}{\sum_{a=1}^{\omega_{0}} m^{d_{a}}}, \ell=1,2, \ldots, \omega_{0}
\end{array}
$$

where, $W_{O \times P}$ represents the weight matrix with $O \times P$ dimension, $e$ denotes the bias, $d_{P \times Q}$ signifies the input feature map with $P \times Q$ dimension, $\varepsilon$ implies the softmax function, $d_{l}$ specifies the element of output layer, and $\omega_{0}$ indicates the output dimension. Figure 3 presents the architectural representation of DRN.

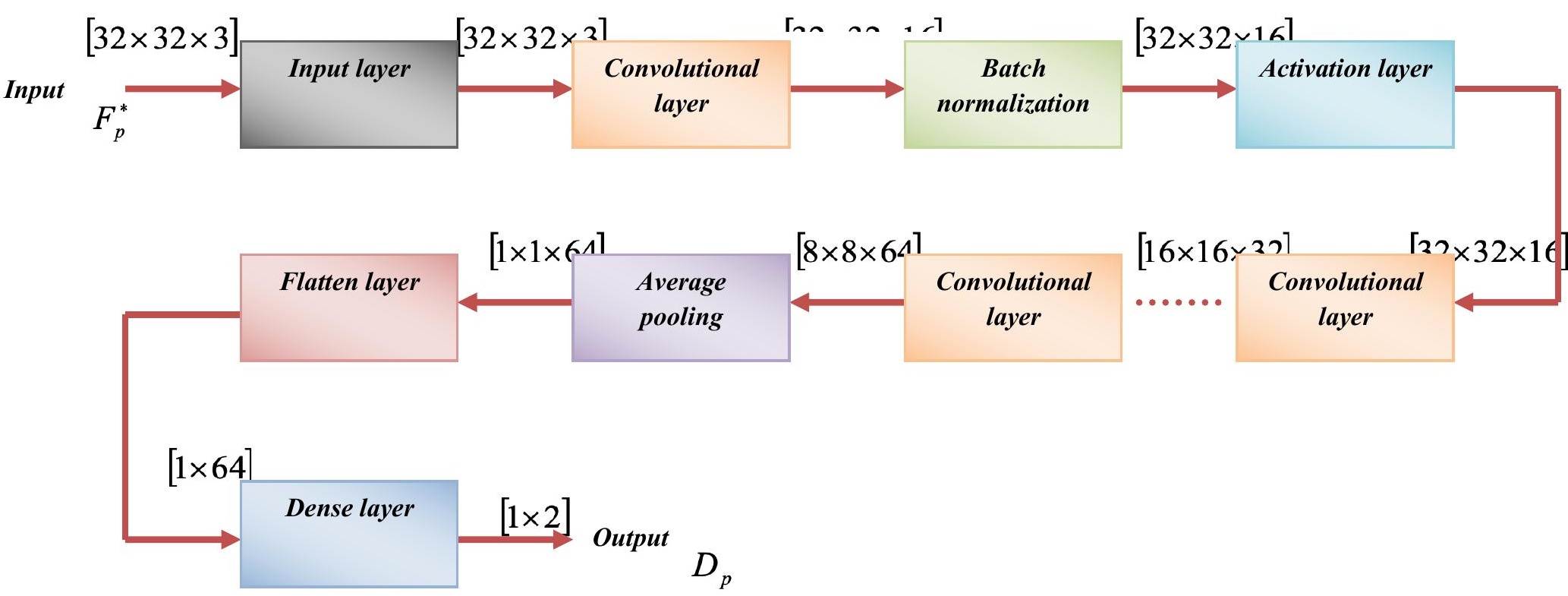

Figure 3. Architecture of DRN

\subsubsection{Training process of DRN using proposed FrWCSO}

The DRN model is trained using the proposed optimization algorithm, named FrWCSO. However, the developed FrWCSO is designed by the combination of FC [10] and WCSO which is the hybridization of WCA [11] and CSO [12], respectively. Furthermore, the fitness equation employed for the DRN classifier is expressed below as, 


$$
\xi=\frac{1}{\delta} \sum_{\tau=1}^{\delta}\left[O-D_{p}\right]^{2}
$$

where, the fitness measure is represented as $\xi, \ell$ implies the overall training samples, the output of DRN classifier is denoted as $D_{p}$ and $O$ specifies the target output. The algorithmic phases of developed FrWCSO algorithm are illustrated in the above section 3.5.Thus, the proposed FrWCSO-based DRN method of pulmonary abnormality detection is very effective in detecting the pulmonary abnormalities as normal and abnormal, which is then offered to the applications for the performance enhancement.

\section{Results and Discussion}

The implementation results of the proposed FrWCSO-based DRN considering the evaluation metrics, such as TPR, TNR, and testing accuracy is presented in this section.

\subsection{Experimental set-up}

The developed FrWCSO-based DRN is implemented in a PYTHON tool using International Conference in Biomedical and Health Informatics (ICBHI 2017) (dataset-1) [24] and respiratory sound database (dataset-2) [25] with the PC having Intel i3 core processor, 2GB RAM, and windows 10 OS.

\subsection{Dataset description}

This section explains the two different datasets utilized for the pulmonary abnormality detection.

Dataset1: This dataset-1 [24] includes the total recording time of 5.5 hours with 6898 respiratory phases, of which 886 have wheezes, 1864 have crackles, and 506 enclose crackles and wheezes in 920 annotated audio samples from different 126 subjects. Meanwhile, cycles are annotated by respiratory experts containing wheezes, crackles, and a mix of wheezes and crackles, or without anomalous respiratory sounds. In addition, heterogeneous equipments are utilized for gathering the signals with the duration of $10 \mathrm{~s}$ to $90 \mathrm{~s}$. Moreover, the exact location of the chest from where the recordings are composed is also provided. Furthermore, the file names are classified into 5 various elements, such as recording index, chest location, patient number, recording equipment, and acquisition mode are separated with underscores $\left(\_\right.$).

Dataset2: This dataset-2 [25] was introduced by two research teams from Greece and Portugal. It comprises 920 annotated recordings of duration from 10s to $90 \mathrm{~s}$. In addition, these recordings are taken from 126 patients. Moreover, the total time of the recording is about 5.5 hours with 6898 respiratory phases of which 1864 have crackles, 886 have wheezes, and 506 enclose wheezes and crackles altogether. The data includes noisy recordings and clean respiratory sounds that emulate real life conditions. The patients cover different groups of age, like elderly, adults and children.

\subsection{Evaluation metrics}

The performance of the proposed FrWCSO-based DRN is analyzed based on the evaluation measures, like TPR, TNR, and testing accuracy.

a) $T P R$ 
It is a measure which calculates the true positive results of detected abnormalities and is expressed as,

$$
\kappa=\frac{a}{a+p}
$$

\section{b) $T N R$}

TNR is a measure which computes the true negative results of detected abnormalities and is formulated as,

$$
\lambda=\frac{b}{b+q}
$$

\section{c) Testing accuracy}

Testing accuracy is a measure which calculates the nearness or correctness of detected abnormalities and the equation is expressed as,

$$
\gamma=\frac{a+b}{a+b+p+q}
$$

where, $a$ denotes true positives, $b$ represents true negatives, $p$ and $q$ implies false positives, and false negatives.

\subsection{Experimental results}

Figure 4) presents the implementation outcomes of developed FrWCSO-based DRN method. The input sound wave is depicted in figure 4 a), the pre-processed sound wave is shown in figure $4 b$ ), the spectral centroid feature output, and the spectral flux feature output is presented in figure $4 c$ ) and $4 d$ ).

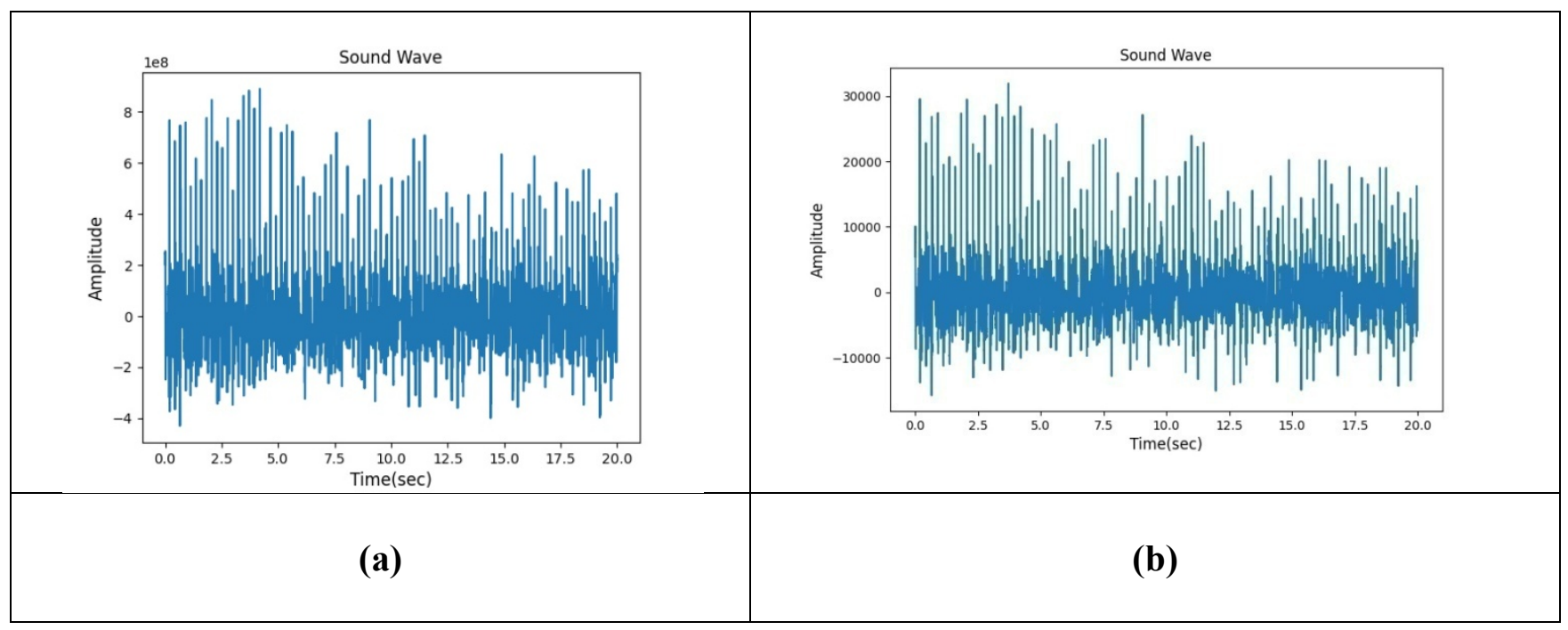




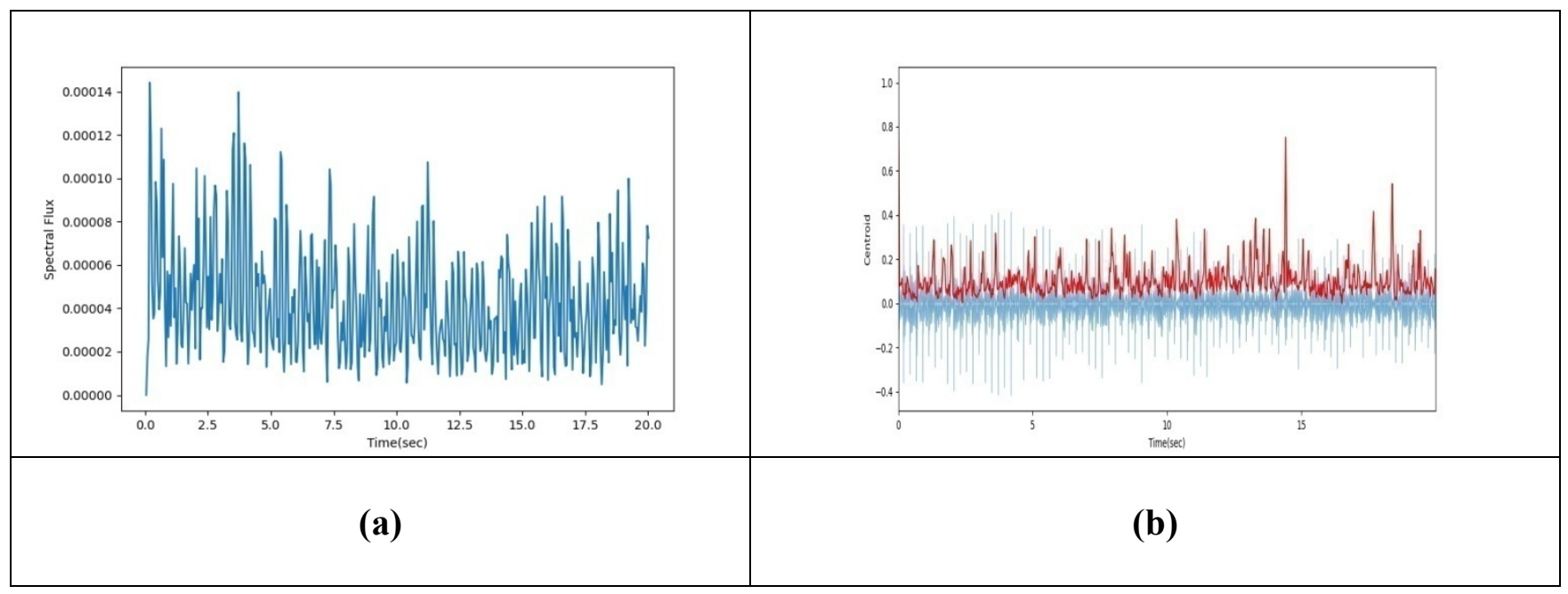

Figure 4. Experimental results: a) input sound wave, b) pre-processed sound wave c) spectral flux feature output d) spectral centroid feature output

\subsection{Performance analysis}

This section elucidates the performance assessment of the developed FrWCSO-enabled DRN method using dataset- 1 and dataset- 2 considering the evaluation metrics, such as TPR, TNR, and accuracy.

\section{(i) Analysis based on dataset 1}

Figure 5 elucidates the performance assessment of the developed method based on dataset-1 by considering the evaluation measures, such as TPR, TNR, and testing accuracy. Figure 5a) presents the analysis based on TPR. With the training data $60 \%$, the TPR value computed by the developed FrWCSO-based DRN with iteration 10 is 0.910 , iteration 20 is 0.913 , iteration 30 is 0.916 , and iteration 40 is 0.919 . The assessment based on TNR is shown in figure $5 \mathrm{~b}$ ). By considering the training data as $70 \%$, the developed FrWCSO-based DRN measured a TNR with iteration 10 is 0.883 , iteration 20 is 0.886 , iteration 30 is 0.890 , and iteration 40 is 0.893 . The analysis using testing accuracy is portrayed in figure $5 \mathrm{c}$ ). Considering $80 \%$ training data, the testing accuracy value computed by the developed FrWCSO-based DRN with iteration 10 is 0.923 , iteration 20 is 0.926 , iteration 30 is 0.930 , and iteration 40 is 0.933 . 


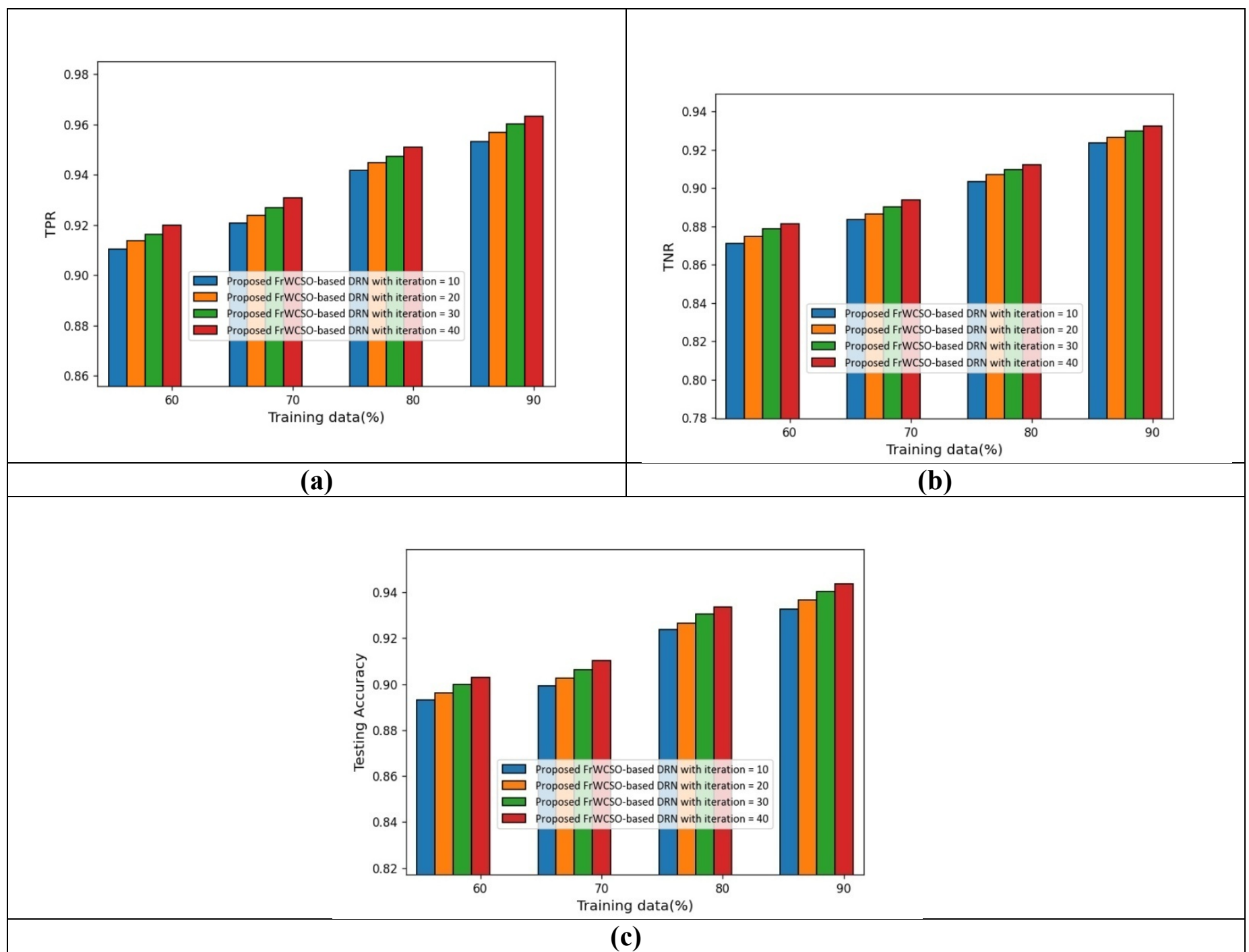

Figure 5.Performance assessment of the developed method using dataset-1 considering a) TPR, b) TNR, and c) Testing accuracy

\section{(ii) Analysis based on dataset-2}

The performance analysis of the developed method using dataset- 2 based on the evaluation measures is shown in figure 6. The assessment using TPR measure is depicted in figure 6a). By considering the training data as 60\%, the developed FrWCSO-based DRN measured a TPR with iteration 10 , iteration 20 , iteration 30 , and iteration 40 is $0.921,0.925,0.928$, and 0.931. Figure 6b) presents the assessment based on TNR. With the training data $70 \%$, the TNR value computed by the developed FrWCSO-based DRN with iteration 10 is 0.873 , iteration 20 is 0.876 , iteration 30 is 0.880 , and iteration 40 is 0.883 . The analysis using testing accuracy is portrayed in figure $6 \mathrm{c}$ ). When the training data is $80 \%$, the testing accuracy value computed by the developed FrWCSO-based DRN with iteration 10 is 0.916 , iteration 20 is 0.919 , iteration 30 is 0.922 , and iteration 40 is 0.925 . 


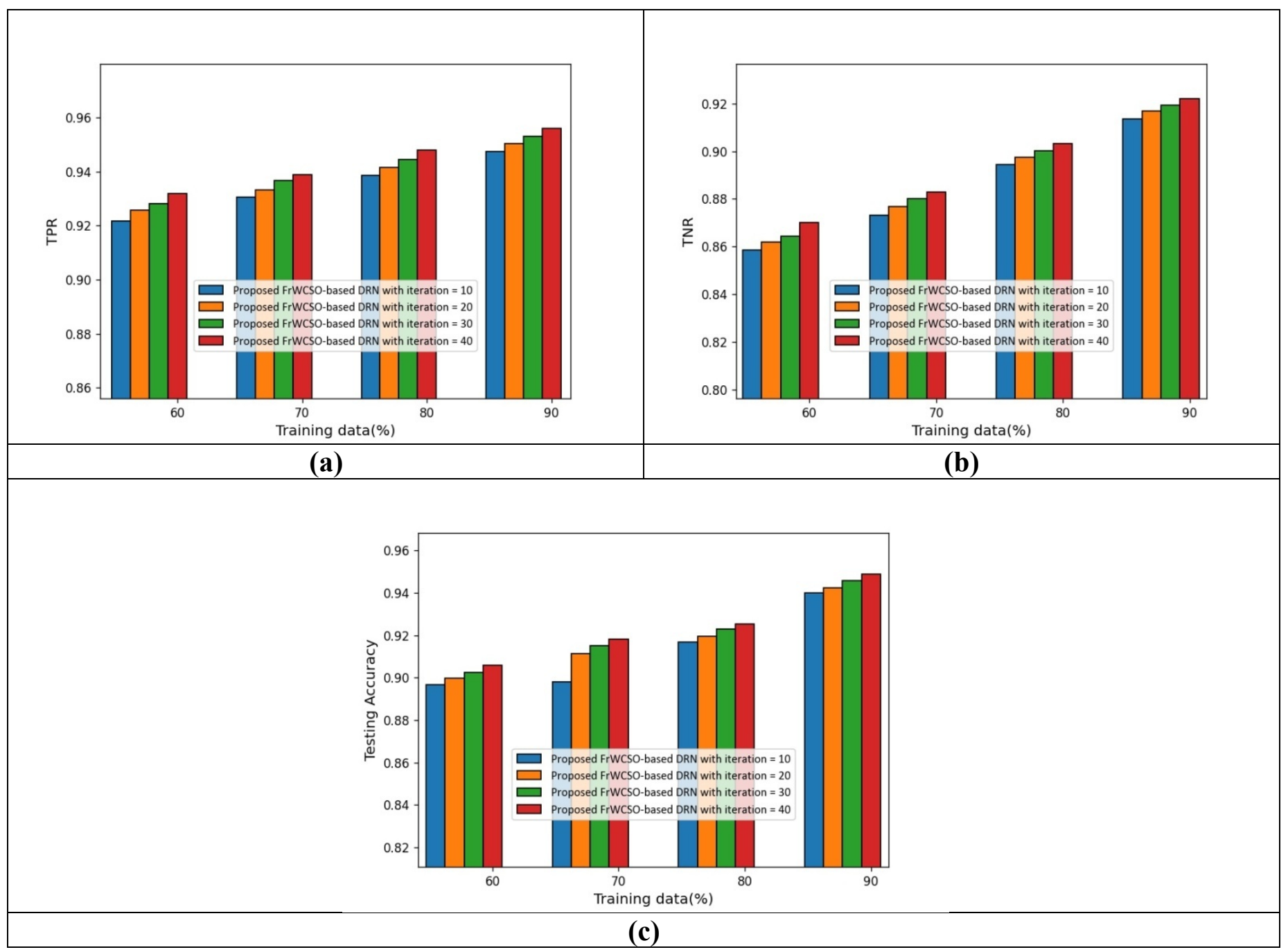

Figure 6.Performance assessment of the developed technique using dataset- 2 considering a) TPR, b) TNR, and c) Testing accuracy

\subsection{Comparative techniques}

The various comparative techniques taken for the assessment are Random Forest classifier [1], Machine Learning [2], DNN [3], CNN [4], WCSO-based HAN, and developed FrWCSO-based DRN.

\subsection{Comparative analysis}

This section elucidates the comparative assessment of the developed technique using dataset1 and dataset-2 based on the evaluation measures such as TPR, TNR, and testing accuracy.

\section{(i) Analysis using dataset-1}

Figure 7 portrays the comparative assessment based on dataset- 1 with respect to TPR, TNR, and testing accuracy metrics. Figure 7a) represents the analysis using TPR. For the $70 \%$ TPR, the value of TPR measured by the developed FrWCSO-based DRN is 0.930 , while the value of TPR measured by the existing techniques, such as Random Forest classifier, machine 
learning, DNN, CNN, and WCSO-based HAN is $0.751,0.819,0.858,0.872$, and 0.912. The performance gain measured by the developed method in comparison with the existing methods is $19.307 \%, 11.938 \%, 7.750 \%, 6.321 \%$, and $2.008 \%$. The assessment based on TNR is portrayed in figure $7 \mathrm{~b}$ ). When the training data is $80 \%$, the developed FrWCSO-based DRN computed a TNR value 0.912 , while the TNR value computed by the existing methods, such as Random Forest classifier is 0.718 , machine learning is $0.771, \mathrm{DNN}$ is $0.809, \mathrm{CNN}$ is 0.841 and WCSO-based HAN is 0.893 . The performance improvement achieved by the developed method in comparison with the existing methods is $21.265 \%, 15.400 \%, 11.248 \%$, $7.705 \%$, and 2.066. Figure 7c) depicts the assessment with respect to testing accuracy. With the training data $60 \%$, the testing accuracy value measured by the Random Forest classifier is 0.710 , machine learning is 0.755 , DNN is $0.803, \mathrm{CNN}$ is 0.847 , WCSO-based HAN is 0.883 , and developed FrWCSO-based DRN is 0.902 . The performance gain measured by the developed method in comparison with the existing methods is $21.340 \%, 16.359 \%, 11.042 \%$, $6.171 \%$, and $2.177 \%$.

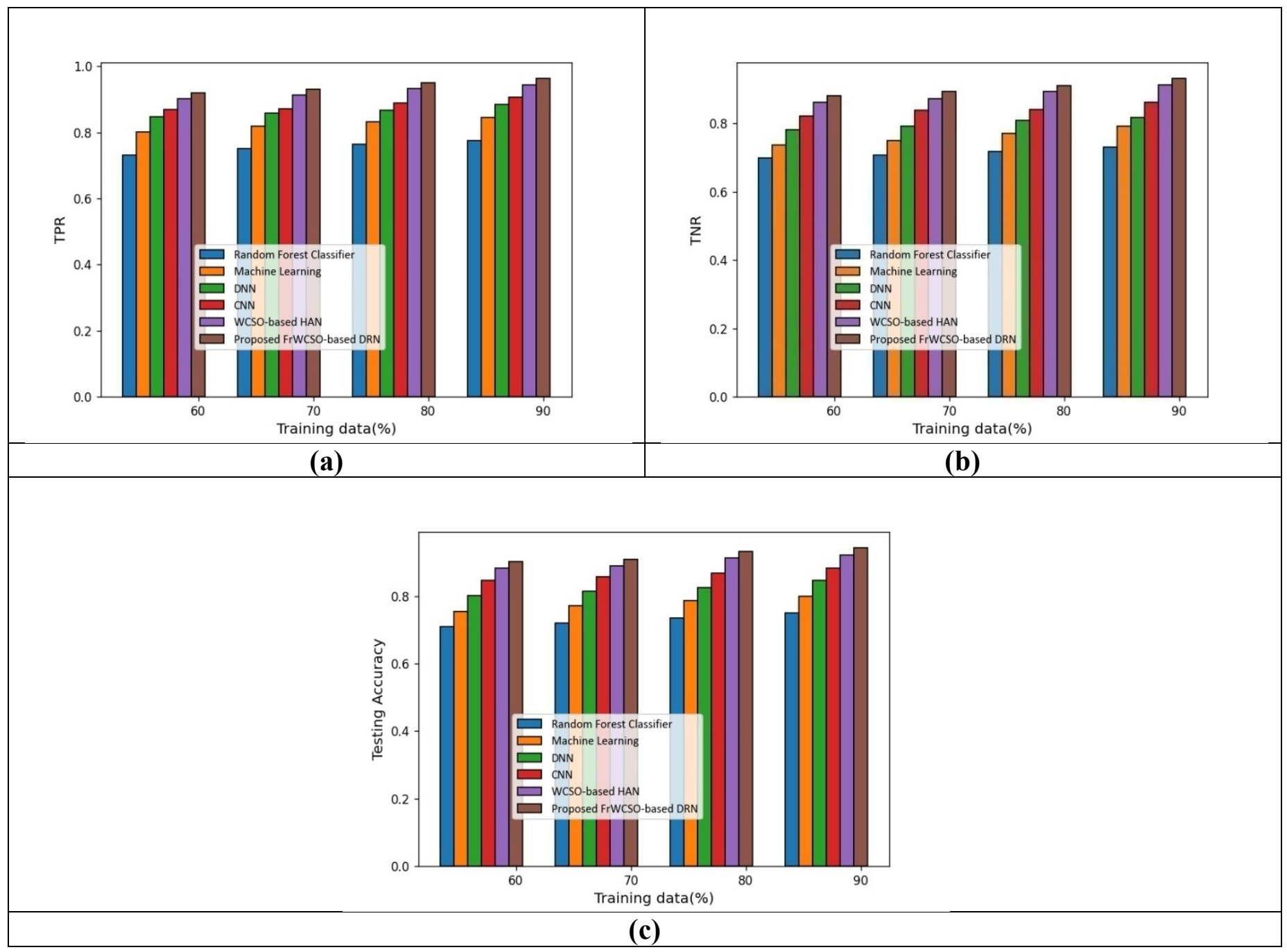

Figure 7.Assessment of the developed method using dataset-1 a) TPR, b) TNR, and c) Testing accuracy 


\section{(ii) Analysis based on dataset-2}

Figure 8 presents the comparative assessment using dataset- 2 by considering the evaluation measures, such as TPR, TNR, and testing accuracy. The assessment based on TPR is presented in figure 8a). When the training data is $60 \%$, the developed FrWCSO-based DRN computed a TPR value of 0.931 , while the TPR value computed by the existing methods, such as Random Forest classifier is 0.743 , machine learning is $0.782, \mathrm{DNN}$ is $0.830, \mathrm{CNN}$ is 0.877 , and WCSO-based HAN is 0.912 . The performance improvement achieved by the developed method in comparison with the existing methods is $20.238 \%, 16.031 \%, 10.913 \%$, $5.866 \%$, and $2.080 \%$. Figure $8 \mathrm{~b}$ ) represents the analysis using TNR. For the $70 \%$ TNR, the value of TNR measured by the developed FrWCSO-based DRN is 0.883 , while the value of TNR measured by the existing techniques, like Random Forest classifier, machine learning, DNN, CNN, and WCSO-based HAN is 0.704, 0.742, 0.807, 0.842, and 0.863. The performance gain measured by the developed method in comparison with the existing methods is $20.258 \%, 15.869 \%, 8.550 \%, 4.632 \%$, and $2.158 \%$. Figure $8 \mathrm{c}$ ) depicts the analysis using accuracy. With the training data $80 \%$, the testing accuracy value measured by the Random Forest classifier is 0.742 , machine learning is 0.787 , DNN is $0.834, \mathrm{CNN}$ is 0.876 , WCSO-based HAN is 0.906 , and developed FrWCSO-based DRN is 0.925 . The performance gain measured by the developed method in comparison with the existing methods is $19.773 \%, 14.899 \%, 9.819 \%, 5.294 \%$, and $2.098 \%$.

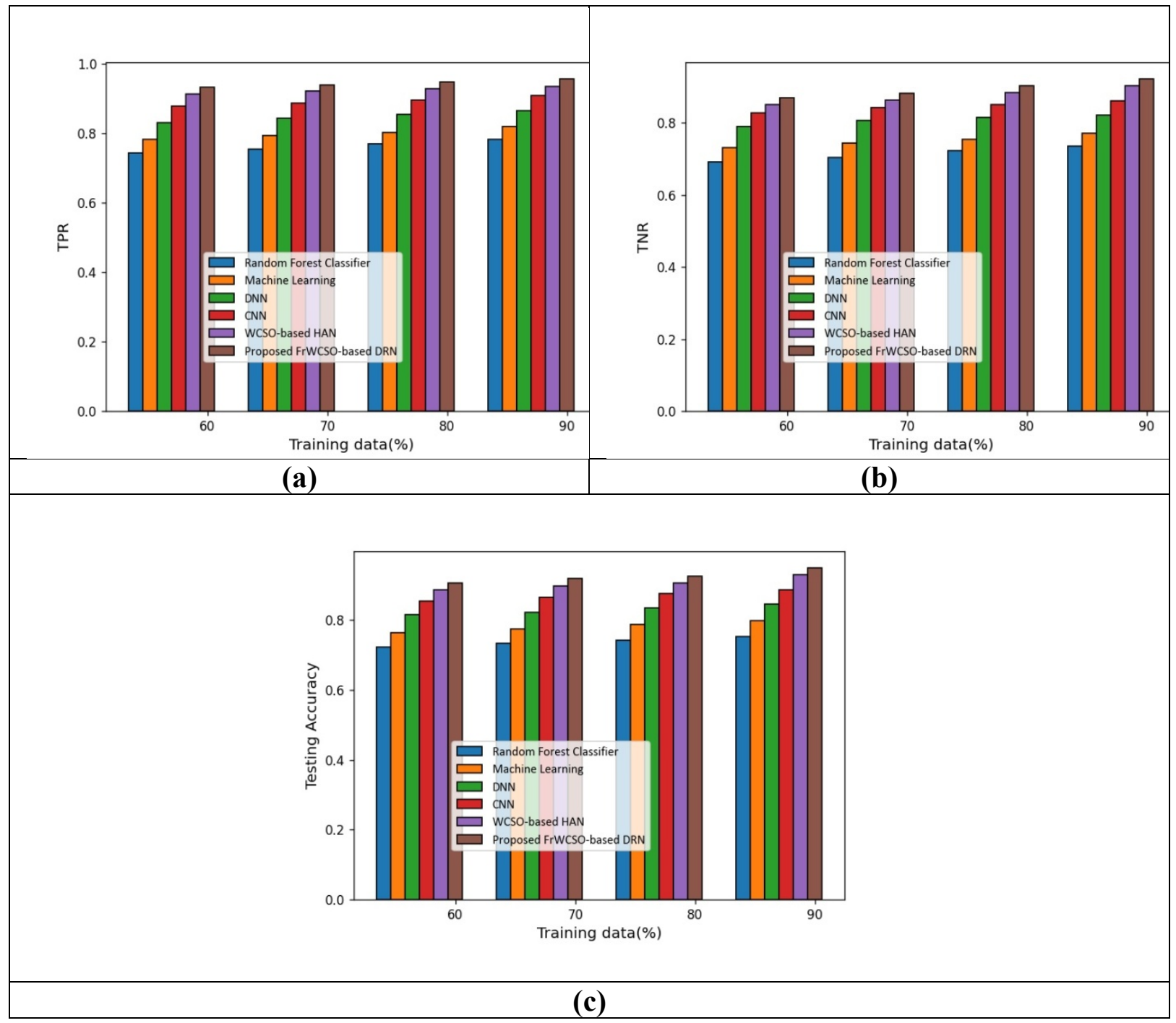


Figure 8. Assessment of the developed method using dataset 2 considering a) TPR b) TNR

c) Testing accuracy

\subsection{Comparative discussion}

Table 2 presents the comparative discussion of the developed FrWCSO-enabled DRN technique in comparison with the existing techniques using the evaluation metrics based on training data $90 \%$. The value of TPR measured by the Random Forest classifier is 0.775 , machine learning is $0.845, \mathrm{DNN}$ is 0.883 , CNN is 0.906 , WCSO-based HAN is 0.943 , and developed FrWCSO-based DRN is 0.963. Likewise, the developed FrWCSO-based DRN computed a TNR value of 0.932, whereas the TNR value measured by the existing approaches, such as Random Forest classifier, machine learning, DNN, CNN, and WCSObased HAN is $0.731,0.791,0.817,0.862$, and 0.913 . Moreover, the testing accuracy value achieved by the Random Forest classifier, machine learning, DNN, CNN, WCSO-based HAN, and developed FrWCSO-based DRN is 0.753, 0.797, 0.844, 0.887, 0.929, and 0.948. From table 2, it is clearly shown that the developed FrWCSO-based DRN computed a higher TPR of 0.963 , higher TNR of 0.932 using dataset-1, and higher testing accuracy of 0.948 using dataset-2, respectively.

\begin{tabular}{|c|c|c|c|c|c|c|c|}
\hline & Metrics & $\begin{array}{c}\text { Random } \\
\text { Forest } \\
\text { classifier }\end{array}$ & $\begin{array}{c}\text { Machine } \\
\text { Learnin } \\
\mathbf{g}\end{array}$ & DNN & CNN & $\begin{array}{c}\text { WCSO-based } \\
\text { HAN }\end{array}$ & $\begin{array}{c}\text { Proposed } \\
\text { FrWCSO-based } \\
\text { DRN }\end{array}$ \\
\hline \multirow{2}{*}{ Dataset 1 } & $\boldsymbol{T P R}$ & 0.775 & 0.845 & 0.883 & 0.906 & 0.943 & $\mathbf{0 . 9 6 3}$ \\
\cline { 2 - 8 } & $\boldsymbol{T N R}$ & 0.731 & 0.791 & 0.817 & 0.862 & 0.913 & $\mathbf{0 . 9 3 2}$ \\
\cline { 2 - 8 } & $\begin{array}{c}\text { Testing } \\
\text { accuracy }\end{array}$ & 0.751 & 0.800 & 0.847 & 0.882 & 0.923 & $\mathbf{0 . 9 4 3}$ \\
\hline Dataset 2 & $\boldsymbol{T P R}$ & 0.781 & 0.81939 & 0.864 & 0.908 & 0.935 & $\mathbf{0 . 9 5 6}$ \\
\cline { 2 - 8 } & $\boldsymbol{T N R}$ & 0.735 & 0.770 & 0.822 & 0.861 & 0.902 & $\mathbf{0 . 9 2 1}$ \\
\cline { 2 - 8 } & $\begin{array}{c}\text { Testing } \\
\text { accuracy }\end{array}$ & 0.753 & 0.797 & 0.844 & 0.887 & 0.929 & $\mathbf{0 . 9 4 8}$ \\
\hline
\end{tabular}

Table2. Comparative discussion of the developed FrWCSO-based DRN technique

\section{Conclusion}

This research presents a novel pulmonary abnormality detection model to detect the pulmonary abnormalities using proposed optimization-enabled deep learning method, called FrWCSO-based DRN. Here, the necessary features, like BFCC, short-term features like spectral flux, spectral centroid, PSD, statistical features like mean, standard deviation, entropy, energy, kurtosis and wavelet transform features are effectively extracted from the respiratory sound signal. After extracting the significant features, data augmentation is done for minimizing the over fitting problems. The proposed FrWCSO is employed for selecting the significant features needed for further processing. Moreover, DRN classifier is accomplished for detecting the pulmonary abnormalities such that the training practice of DRN is done using the developed optimization algorithm, named FrWCSO, which is newly designed by the integration of FC and WCSO. Meanwhile, WCSO is the combination of WCA and CSO. Furthermore, the developed FrWCSO-based DRN outperformed various existing techniques and achieved effective performance in terms of TPR, TNR, and testing 
accuracy with the higher values of $0.963,0.932$, and 0.948 . The future work would be the consideration of devising larger datasets to improve the detection performance.

\section{Ethics approval and consent to participate}

Approved by RDC of mansarovar Global University Madhya Pradesh India

\section{Consent for publication}

Yes

8.Availability of data and materials

Not applicable

\section{Competing interests}

No conflict of interest exists. We wish to confirm that there are no known conflicts of interest associated with this publication and there has been no significant financial support for this work that could have influenced its outcome.

\section{Funding}

Not applicable

\section{Authors' contributions}

(a).Conception and design of study: all authors contributed

(b).acquisition of data: all authors contributed

(c).analysis and interpretation of data: all authors contributed

(d).drafting of manuscript: all authors contributed

(e).Approval of the version of the manuscript to be published: all authors consent

\section{Acknowledgements}

All persons who have made substantial contributions to the work reported in the manuscript (e.g technical help,writing and editing assistance,general support.) 


\section{References}

[1] Alfonso Monaco, Nicola Amoroso, Loredana Bellantuono, Ester Pantaleo, Sabina Tangaro, and Roberto Bellotti, "Multi-Time-Scale Features for Accurate Respiratory Sound Classification", Applied Sciences, vol.10, no.23, pp.8606, 2020.

[2] Fei Meng, Yan Shi, Na Wang, Maolin Cai, and Zujing Luo, "Detection of Respiratory Sounds Based on Wavelet Coefficients and Machine Learning", IEEE Access, vol.8, pp.155710-155720, 2020.

[3] JyotibdhaAcharyay, and Arindam Basuyyy, "Deep Neural Network for Respiratory Sound Classification in Wearable Devices Enabled by Patient Specific Model Tuning", IEEE transactions on biomedical circuits and systems, vol.14, no.3, pp.535-544, 2020.

[4] Samiul Based Shuvo, Shams Nafisa Ali, Soham Irtiza Swapnil, Taufiq Hasan, and Mohammed Imamul Hassan Bhuiyan2, "A lightweight CNN model for detecting respiratory diseases from lung auscultation sounds using emd-cwt-based hybrid scalogram", IEEE Journal of Biomedical and Health Informatics, 2020.

[5] Fraiwan M, Fraiwan L, Alkhodari M, Hassanin O., "Recognition of pulmonary diseases from lung sounds using convolutional neural networks and long short-term memory", Journal of Ambient Intelligence and Humanized Computing, pp.1-3, April 2021.

[6] Baghel N, Nangia V, Dutta MK., "ALSD-Net: Automatic lung sounds diagnosis network from pulmonary signals", Neural Computing and Applications, pp.1-6, July 2021.

[7] Khan SI, Palodiya V, Poluboyina L., "Automated classification of human lung sound signals using phase space representation of intrinsic mode function.

[8] Jayalakshmy S, Sudha GF., "Scalogram based prediction model for respiratory disorders using optimized convolutional neural networks", Artificial intelligence in medicine, vol.103, pp.101809, March 2020.

[9] Zhicong Chen, Yixiang Chen, Lijun Wu, Shuying Cheng, and PeijieLin, "Deep residual network based fault detection and diagnosis of photovoltaicarrays using current-voltage curves and ambient conditions", Energy Conversion and Management, vol.198, no.111793, 2019.

[10] Pawan R. Bhaladhare1 and Devesh C. Jinwala, "A Clustering Approach for the $l$ Diversity Model in PrivacyPreserving Data Mining Using Fractional Calculus-Bacterial", Advances in Computer Engineering, 2014.

[11] HadiEskandar, Ali Sadollah, ArdeshirBahreininejad, and Mohd Hamdi, "Water cycle algorithm-A novel metaheuristic optimization method for solving constrained engineering optimization problems", Computers \& Structures, vol.110, pp.151-166, 2012.

[12] Ran Cheng and YaochuJin, "A competitive swarm optimizer for large scale optimization", IEEE transactions on cybernetics, vol.45, no.2, pp.191-204, 2014.

[13] Haridas, A.V., Marimuthu, R. and Chakraborty, B., "A novel approach to improve the speech intelligibility using fractional delta-amplitude modulation spectrogram", Cybernetics and Systems, vol.49, no.7-8, pp.421-451, 2018. 
[14] Spectral gating based noise reduction technique "https://timsainburg.com/noisereduction-python.html”, accessed on June 2021.

[15] Kumar, C., Ur Rehman, F., Kumar, S., Mehmood, A. and Shabir, G., "Analysis of MFCC and BFCC in a speaker identification system", In proceedings of International Conference on Computing, Mathematics and Engineering Technologies (iCoMET), pp.1-5, March 2018.

[16] Alsolamy M, Fattouh A., "Emotion estimation from EEG signals during listening to Quran using PSD features", In 2016 7th International Conference on Computer Science and Information Technology (CSIT), pp.1-5, July 2016.

[17] Aliyu I, Lim CG., "Selection of optimal wavelet features for epileptic EEG signal classification with LSTM", Neural Computing and Applications, pp.1-21, January 2021.

[18] Pan Q, Li X, Fang L., "Data Augmentation for Deep Learning-Based ECG Analysis", In Feature Engineering and Computational Intelligence in ECG Monitoring, pp.91-111, 2020.

[19] Sovijarvi AR., "Characteristics of breath sounds and adventitious respiratory sounds", Eur Respir Rev, vol.10, pp.591-6, 2000.

[20] Lee SJ, Kim SW, Kong KA, Ryu YJ, Lee JH, Chang JH., "Risk factors for chronic obstructive pulmonary disease among never-smokers in Korea", International journal of chronic obstructive pulmonary disease, vol.10 no.497, 2015.

[21] Khan TA, Vijayakumar P., "Separating Heart Sound from Lung Sound Using Lab VIEW", International Journal of Computer and Electrical Engineering, vol.2, no.3, pp.524, June 2010.

[22] Koul, P. A., "Chronic obstructive pulmonary disease: Indian guidelines and the road ahead”, Lung India, vol.30, no.3, pp.175-177, 2013.

[23] Sarkar M, Madabhavi I, Niranjan N, Dogra M., "Auscultation of the respiratory system", Annals of thoracic medicine, vol.10, no.3, pp.158, July 2015.

[24] ICBHI 2017 challenge database, "https://bhichallenge.med.auth.gr/", accessed on September 2021.

[25] Respiratory Sound Database, "https://www.kaggle.com/vbookshelf/respiratory-sounddatabase", accessed on September 2021.

[26] Yadav A, Dutta MK, Prinosil J., "Machine Learning Based Automatic Classification of Respiratory Signals using Wavelet Transform", In 2020 43rd International Conference on Telecommunications and Signal Processing (TSP), pp.545-549, July 2020.

[27] Yan Shi, Yuqian Li, Maolin Cai, Xiaohua Douglas Zhang, "A lung sound category recognition method based on wavelet decomposition and BP neural network", International journal of biological sciences, vol.15, no.1, pp.195, 2019.

[28] Sengupta N, Sahidullah M, Saha G., "Lung sound classification using cepstral-based statistical features", Computers in biology and medicine, vol.75, pp.118-29, August 2016.

[29] Yamashita M, Matsunaga S, Miyahara S., "Discrimination between healthy subjects and patients with pulmonary emphysema by detection of abnormal respiration", In 2011 IEEE 
International Conference on Acoustics, Speech and Signal Processing (ICASSP), pp.693-696, May 2011.

[30] Jin F, Sattar F, Goh DY., "New approaches for spectro-temporal feature extraction with applications to respiratory sound classification", Neurocomputing, vol.123, pp.362-71, January 2014.

\section{Authors' information}

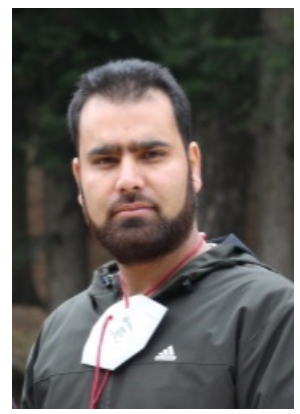

Jawad ahmad dar (Graduate student, received B.Tech degree in CSE from IUST Kashmir and M.Tech degree in CSE from Kurukshetra University Kurukshetra india, he is currently persuing the Ph.D. degree in engineering(CSE) from Mansarovar Global University (MGU) india.He has been involved in varying roles as a research analyst and an Assistant Professor. His current research interests include artificial intelligence, Machine Learning and Deep Learning),designing of cryptographic algorithms.

Email Id: jawadsirphysics@gmail.com

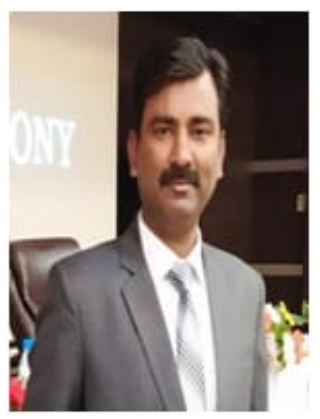

Dr kamal Kr.Srivastava is working as a Professor in faculty of Computer Science and Engineering,Department in Mansarovar Global University Madhya Pradesh.He was also associate with Dr APJ abdul Kalam Technical University Uttar Pradesh. Dr kamal $\mathrm{Kr}$.Srivastava received his Phd degree in field of computer science and Engineering for increasing the efficiency of cloud computing.He has also presented and published many papers at several reputed national and international conferences and journal. Dr kamal Kr.Srivastava is also Reviewer and member of many reputed journal and international conferences.His research activities are currently related with artificial Intelligence,Deep Learning and Machine Learning Email Id: 2007.srivastava@gmail.com

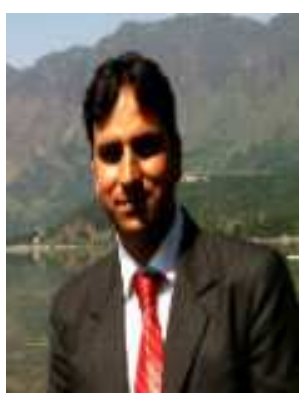

Dr Sajaad Ahmed Lone received the M.Tech in Information Technology from Guru Gobind Singh Indraprastha University New Delhi and $\mathrm{PhD}$ from National Institute of Technology, Srinagar Jammu and Kashmir India. He is currently an Assistant Professor of Computer Science \& Engineering in the Department of Computer Science and Engineering, Islamic University of Science and Technology Jammu and Kashmir, India. His current research interests include Biometrics, Image Processing, Network Security. He has received numerous awards, including the young scientist award, throughout his academic journey. Email Id: sajaad.lone@islamicuniversity.edu.in 


\section{Supplementary Files}

This is a list of supplementary files associated with this preprint. Click to download.

- GraphicalAbstract.docx 